\title{
Trattamento delle ulcere croniche difficili: il ruolo centrale della preparazione del letto della ferita
}

\section{Maria Stella Aliquò ${ }^{1}$, Luca Monge ${ }^{2}$, Fabio Romagnoli ${ }^{3}$, Rolando Tasinato ${ }^{4}$}

\author{
${ }^{1}$ Dipartimento di Medicina Interna, ARNAS Civico, Palermo \\ ${ }^{2}$ AOU Città della Salute e della Scienza di Torino, SSD Gestione Complicanze del Diabete \\ Centro per la Cura del Piede Diabetico Presidio CTO \\ ${ }^{3}$ UOC Centro Piede Diabetico Istituto INRCA IRCCS, Ancona \\ ${ }^{4}$ UO di Chirurgia Generale Ambulatorio di Chirurgia Vascolare Regione Veneto ASL 13, \\ Ospedale di Mirano (Venezia)
}

\section{Introduzione}

Le ulcere croniche rappresentano una sfida continua per gli operatori sanitari impegnati in ambito vulnologico e un problema significativo di salute pubblica con un carico economico importante per il Sistema Sanitario Nazionale.

Stime epidemiologiche condotte a livello internazionale evidenziano che il numero di persone affette da piaghe da decubito, ulcere degli arti inferiori, lesioni del piede in presenza di diabete, deiscenze di ferite traumatiche e/o chirurgiche è elevato.

Negli Stati Uniti ogni anno si contano circa 6,5 milioni di ulcere cutanee croniche da pressione, da stasi venosa e da diabete mellito [1]. Secondo le ultime linee

\section{Indirizzo per la corrispondenza:}

Maria Stella Aliquò

e-mail: ms.aliquo@gmail.com

Accettato: 30/05/2017 - Pubblicato online: 07/07/2017

(C) 2017 The Authors. This article is published by HPS Srl and licensed under Creative Commons Attribution-NC-ND 4.0 International (CC BY-NC-ND 4.0). Any commercial use is not permitted and is subject to Publisher's permissions. Full information is available at www.aboutpharma.com/publishing/riviste/aboutopen/ guida dell'European Dermatology Forum le ulcere dell'arto inferiore interessano dall' $1,5 \%$ al $3 \%$ della popolazione generale e le percentuali arrivano al 4-5\% nei soggetti di età $\geq 80$ anni [2]. I dati sono ancora più elevati per quanto riguarda il piede diabetico: l'incidenza annuale di ulcera diabetica, infatti, oscilla tra $1 ' 1,9 \%$ e il 4,1\%, mentre per la prevalenza l'insorgenza di lesioni ulcerative a carico del piede arriva al 7,7\% (contro il 2,8\% della popolazione generale) e alcuni studi hanno riscontrato nei diabetici una probabilità di sviluppare piede diabetico nell'arco della vita compresa tra il $15 \%$ e il $25 \%$ [3]. Numeriche epidemiologiche di queste dimensioni hanno correlati economici di estrema rilevanza: negli Stati Uniti i costi sanitari dovuti alle ulcere cutanee croniche sono nell'ordine dei 15 miliardi di dollari all'anno [1]. Uno studio effettuato nel Regno Unito (rapporto THIN, The Health Improvement Network, 2012-2013), ha evidenziato che, in termini economici, la gestione delle ulcere cutanee si aggira intorno ai 4-5,1 miliardi di sterline all'anno, un costo paragonabile al budget impiegato per combattere l'obesità in Gran Bretagna, equivalente al $4 \%$ circa dei costi totali del sistema sanitario anglosassone, percentuale destinata a cresce- 
re costantemente con l'invecchiamento della popolazione [4]. Un'analisi condotta dallo stesso gruppo di ricerca ha mostrato che un'ulcera guarita costa al sistema sanitario britannico tra le 698 e le 3.998 sterline per paziente mentre il costo di una mancata guarigione oscilla tra 1.719 e 5.976 sterline a paziente [5]. Questo significa che il costo sanitario per un'ulcera non guarita è in media del 135\% più elevato di quello di una lesione portata a guarigione.

Un altro dato che fa riflettere è emerso dal report dell'analisi del database THIN e riguarda la mancata diagnosi o caratterizzazione dell'ulcera nel 30\% dei pazienti [4]. è evidente un problema di fondo: a fronte di un impatto economico-sanitario di prima grandezza, le conoscenze sul corretto trattamento delle ulcere, in particolare per quello che riguarda la preparazione del letto della ferita, non sono così diffuse come ci si potrebbe e dovrebbe aspettare.

\section{Aspetti centrali del trattamento: il microambiente della ferita}

Le ulcere croniche sono lesioni che non sono riuscite a procedere verso un processo riparativo ordinario e tempestivo d'integrità anatomica e funzionale nei successivi 3 mesi dall'insorgenza [6].

La sequenza fisiologica e dinamica del processo riparativo viene distinta in infiammazione, proliferazione e rimodellamento, e rappresenta un continuum denominato "cascata di guarigione". Le lesioni cutanee che non rispettano questa sequenza, spesso dovuta alle comorbilità presenti, tendono a cronicizzare.

Queste condizioni complesse hanno bisogno di un approccio di cura e riabilitativo di tipo interdisciplinare e specialistico nel tempo $[7,8]$.

Aspetto fondamentale nel trattamento dell'ulcera è la preparazione ottimale del letto della ferita (wound bed preparation, WBP), da intendere come gestione globale e coordinata della lesione, volta ad accelerare i processi endogeni di guarigione ma anche a promuovere l'adozione di misure terapeutiche efficaci [9]. Va sottolineato che il microambiente della ferita cronica è caratterizzato da attività biochimiche, cellula- ri e segnali molecolari alterati che comportano una sovrapposizione e, quindi, un'inefficienza e il blocco del processo riparativo [10]. Il protrarsi della flogosi comporta aumento di citochine proinfiammatorie, alterazione dell'attività mitotica, aumento delle proteasi, senescenza cellulare, alterazione del $\mathrm{pH}$ e formazione di specie reattive dell'ossigeno (ROS). Le interazioni delle attività cellulari e biochimiche sono strettamente collegate tra loro: è riconosciuta la correlazione tra bioburden (carica batterica), attività delle proteasi, equilibrio dei fluidi, pH e ROS. Tutti questi fattori costituiscono gli elementi principali del microambiente della ferita [11].

Le proteasi (conosciute anche come metalloproteinasi, MMP) sono enzimi prodotti da diverse cellule del tessuto connettivo e secreti nella matrice cellulare, che agiscono sulle proteine della matrice extracellulare (MEC) scindendole in peptidi e aminoacidi [12]. Diverse proteasi ferita-correlate agiscono su diverse proteine; fino ad oggi sono state individuate almeno 27 MMP, suddivise in 7 gruppi in funzione del substrato su cui agiscono: (1) collagenasi, (2) gelatinasi, (3) stromelisine, (4) matrilisine, (5) metalloelastasi, (6) MMP di membrana e (7) altre MMP.

Nella fase infiammatoria favoriscono l'eliminazione della MEC danneggiata; nella fase proliferativa promuovono la degradazione della membrana per l'angiogenesi e contribuiscono al distacco e alla migrazione delle cellule, e nella fase di rimodellamento favoriscono la contrazione e il rimodellamento della MEC [12]. Tuttavia, nella ferita cronica il prolungamento della fase flogistica interferisce con la normale guarigione per uno sbilanciamento tra deposizione di tessuto, stimolato dai fattori di crescita, e distruzione del tessuto mediato dalle proteasi. Gli stimoli che possono mantenere alti i livelli di metalloproteasi sono collegati alla presenza di tessuto necrotico, corpi estranei, carica batterica. Le MMP sono legate anche al valore di $\mathrm{pH}$ del microambiente della ferita, valori di $\mathrm{pH}$ alcalino ne favoriscono l'attività [13,14]. Nelle lesioni croniche sono stati rilevati valori alcalini di $\mathrm{pH}$ compresi tra 7,15 e 8,9, motivo per cui l'azione delle MMP si mantiene elevata. Diversamente, nello strato corneo della cute integra il valore di $\mathrm{pH}$ è compreso 
tra 4 e 6. L'ampiezza di questo intervallo nella cute sana è spiegabile sulla base di diversi fattori quali umidità, sudore, sebo, sito anatomico, predisposizione genetica ed età $[15,16]$. L'acidità del manto idrolipidico che riveste la superficie della cute fornisce idratazione, protezione batterica e funzione di barriera agli agenti chimici esterni.

Il mantenimento dell'acidità influisce nel processo di desquamazione delle cellule e sulla crescita della flora batterica residente [17].

Una volta persa l'integrità di questo strato e, mano a mano che si espongono gli strati sottostanti, il pH inizia ad aumentare e diventa basico.

Questo fenomeno ha come conseguenza [13]

- la creazione di un ambiente favorevole alla crescita e proliferazione batterica anche di quelle specie riscontrabili normalmente sulla cute integra che non si moltiplicano grazie al pH acido;

- il favorire un'infiammazione cronica legata all'attivazione di metalloproteasi, enzimi che risultano inattivi solo a valori di $\mathrm{pH}<4$.

Inoltre studi in vitro hanno evidenziato rallentamento della sintesi cellulare e della migrazione cellulare a pH basico [18].

\section{\| ruolo delle infezioni e del biofilm}

La crescita e proliferazione batterica nell'ulcera cronica costituiscono un punto di particolare rilevanza. Nelle lesioni croniche i batteri patogeni più frequentemente isolati sono Gram-positivi, Gram-negativi e anaerobi. Sono state osservate importanti differenze nelle specie microbiche responsabili di infezione a seconda della patogenesi della lesione cronica e va ricordato che la flora microbica di una ferita cronica si modifica nel tempo, tendendo a ridursi la componente microbica e aumentando quella micotica [19]. I dati sperimentali sul ruolo della carica batterica nel determinare l'infezione di una ferita non sono univoci. In presenza di batteri a elevata patogenicità come lo streptococco beta-emolitico sono rilevanti anche basse cariche microbiche, ma in genere l'infezione si sviluppa in presenza di una carica infettante significativa, cioè di una concentrazione di microrganismi superiore a 105 unità formanti colonie (CFU) per grammo di tessuto [20].

La presenza di strati di materiale glicoproteico aderente al letto dell'ulcera, definito "biofilm", costituisce un altro fattore importante nella patogenesi dell'infezione. I biofilm rappresentano colonie batteriche altamente organizzate che permettono ai microrganismi di interagire vicendevolmente scambiandosi nutrienti e metaboliti. I biofilm sono foci protetti di infezione e di resistenza batterica all'interno dell'ulcera, che proteggono i batteri dall'effetto degli agenti antibiotici e antisettici [21].

L'infezione provoca un aumento dell'essudato, la cui composizione è tale da rallentare e bloccare la proliferazione di cellule chiave quali cheratinociti, fibroblasti e cellule endoteliali. Nelle ferite croniche l'essudato contiene concentrazioni elevate di mediatori di infiammazione e di MMP in grado di degradare la matrice delle proteine extracellulari [22]. La componente essudativa, se abbondante, crea squilibrio elettroliti$\mathrm{co}$, altera la funzione barriera della cute perilesionale e comporta un rallentamento nella guarigione della ferita, alterando la qualità di vita del paziente [23].

La detersione del letto della ferita: soluzione acido-ossidante a base di acido ipocloroso

Un passo essenziale e obbligatorio nell'ambito di un efficace trattamento dell'ulcera è rappresentato dalla rimozione del tessuto necrotico e dalla detersione del letto della lesione. Il tessuto necrotico prolunga la risposta infiammatoria, impedisce meccanicamente la contrazione dell'ulcera e ostacola il processo di riepitelizzazione $[24,25]$.

La detersione primaria attraverso il lavaggio della ferita ha lo scopo di rimuovere, con l'allontanamento meccanico, qualsiasi materiale presente nella ferita: detriti, residuo di medicazione, tessuto devitalizzato non aderente. Inoltre, la detersione della ferita comporta una riduzione della carica microbica e di conseguenza la possibile diminuzione di una componente 
infiammatoria del letto della ferita. Quindi la detersione è un requisito indispensabile per la guarigione della ferita, particolarmente per le lesioni cronicizzate. Recentemente, grazie all'introduzione di una soluzione acido-ossidante a base di acido ipocloroso, si è delineato un nuovo concetto di detersione attiva ovvero l'affiancamento di un trattamento dinamico atto a bilanciare gli elementi che regolano il microambiente.

La scelta della soluzione acido-ossidante a base di acido ipocloroso, insieme a una soluzione antisettica di provata efficacia come il sodio ipoclorito allo $0,05 \%$, rappresenta il binomio innovativo di prevenzione/trattamento di ferite infette, in tutte le condizioni in cui si rende necessario potenziare l'attività di controllo della carica batterica.

Vengono considerati a rischio i pazienti affetti da diabete, patologie vascolari arteriose non compensate da un sufficiente apporto ematico o dalla terapia compressiva (ulcere venose), pazienti immunodepressi, insufficienze d'organo, malnutrizione, obesità, età avanzata.

La soluzione acido-ossidante contiene cloro libero di cui oltre il 95\% è presente sotto forma di acido ipocloroso $(\mathrm{HClO})$ ad alta purezza, con $\mathrm{pH}$ compreso fra 2,5 e 3,0 ed alto potenziale di ossidoriduzione.

L'acido ipocloroso favorisce la riduzione del bioburden (carica batterica), il pH acido $<3,0$ crea un ambiente sfavorevole alla vita dei microrganismi e ne riduce la crescita, l'elevato potere di ossidoriduzione danneggia fisicamente la membrana cellulare dei batteri. La combinazione di $\mathrm{pH}$ basso e potenziale di ossidoriduzione elevato consente di inibire le metalloproteasi della matrice.

Le esperienze di utilizzo nella pratica clinica documentano l'efficacia della soluzione acido-ossidante a base di acido ipocloroso nel promuovere la guarigione di lesioni ulcerose di diversa natura, come piede diabetico, ulcere flebopatiche e da pressione.

Viene di seguito presentata una raccolta di casi clinici particolarmente significativi, gentilmente concessi dai dottori Maria Stella Aliquò, Luca Monge, Fabio Romagnoli e Rolando Tasinato.

\section{Caso clinico 1 - Ulcera neuro-ischemica} con fascite in corso di diabete

Maria Stella Aliquò

Presentazione clinica - Il paziente, diabetico, giunge all'osservazione in ambulatorio, dopo essere stato precedentemente seguito in altro nosocomio; presenta ulcere necrotiche del II e V metatarso destro con abbondante essudato verdastro (grado 3D secondo la Texas Classification) [Figura 1].

Dati anagrafici - Maschio, 57 anni.

Anamnesi patologica remota - Il paziente è affetto da anni da ipertensione arteriosa; diabete mellito di tipo 2 complicato con neuropatia diabetica; arteriopatia obliterante degli arti inferiori

Esame obiettivo della lesione - Si evidenza un'ulcera neuro-ischemica con infiltrazione della fascia plantare e presenza di fascite.

Indagini di approfondimento diagnostico - Gli esami ematochimici indicano insufficienza renale (clearance della creatinina $38 \mathrm{ml} / \mathrm{min}$ ), insufficiente controllo glico-metabolico (HbA1c 10,1\%) e aumento degli indici di flogosi (PCR 12,1 mg/dl). L'ecodoppler degli arti inferiori mostra una diffusa ateromasia; buono il flusso in assenza di stenosi emodinamicamente significative a sinistra, a destra femorale superficiale chiusa all'origine, flusso popliteo tibiale distalmente molto ridotto.

Trattamento - Lavaggio del piede con soluzione per igiene intima a $\mathrm{pH}$ acido. Il paziente è quindi sottoposto a debridement chirurgico e amputazione del V dito. Un frammento osseo è inviato per la coltura batterica e in attesa del risultato si prescrive una terapia antibiotica empirica.

Decorso - Regolare, con visita dopo 3 giorni.

Gestione chirurgica - Si effettua l'incisione e il drenaggio dell'ascesso plantare, oltre al lavaggio con soluzione di sodio ipoclorito allo $0,05 \%$, lasciando aperta la ferita [Figura 2]. 

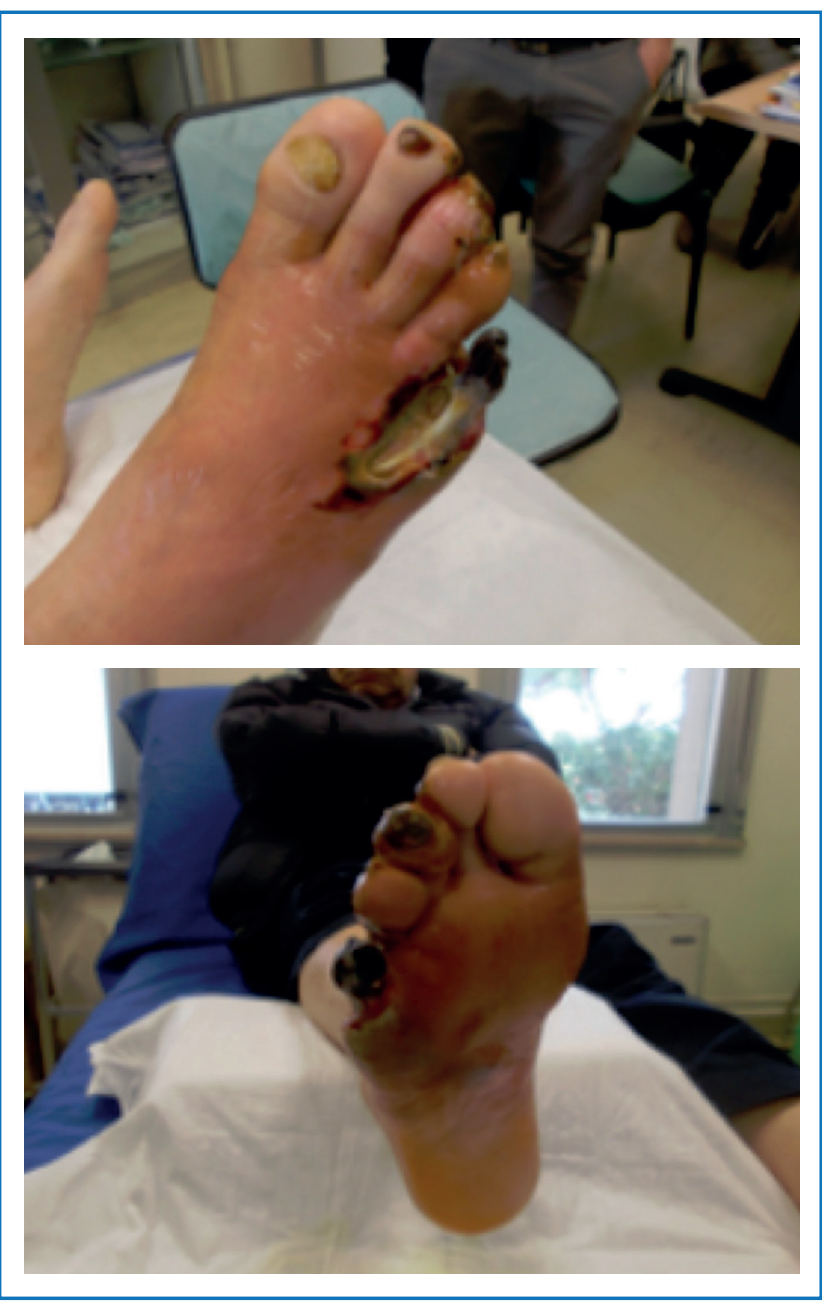

Figura 1. II paziente alla prima osservazione.

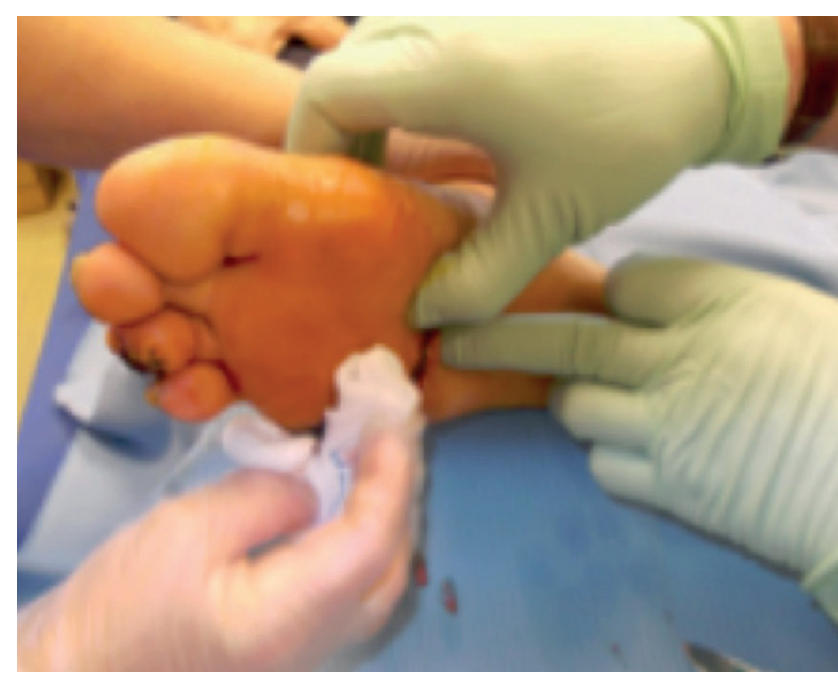

Figura 2. Incisione della fascia plantare e drenaggio.
Decorso - Regolare; il paziente torna al controllo dopo una settimana.

Trattamento - Si procede a una sutura parziale con nuovo lavaggio [Figura 3]. Data l'esposizione della testa metatarsale, si applica la vacuumterapia (pressione negativa $120 \mathrm{mmHg}, 5$ applicazioni a giorni alterni) [Figura 4].

Decorso - Programmati controlli ambulatoriali settimanali ed eseguita angioplastica percutanea (PTA).

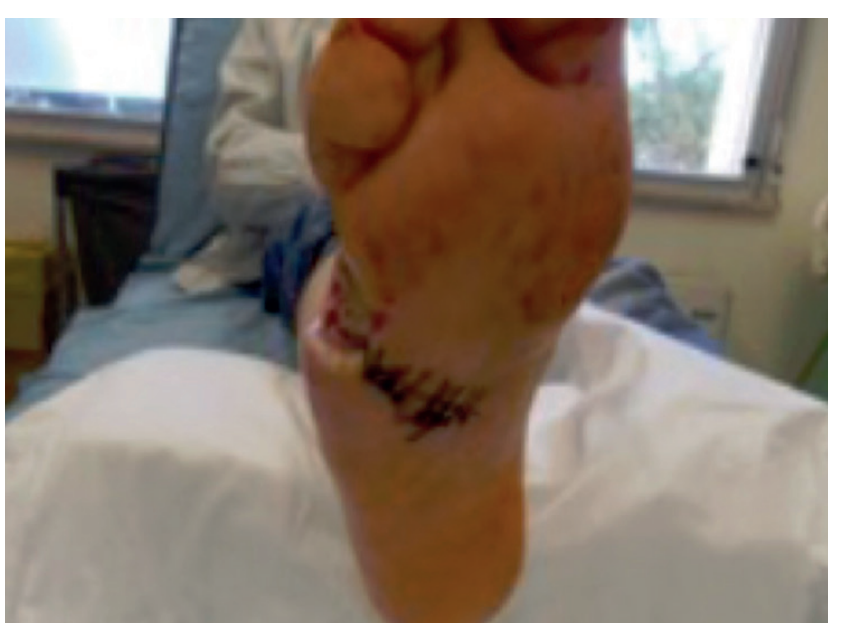

Figura 3. Sutura parziale.

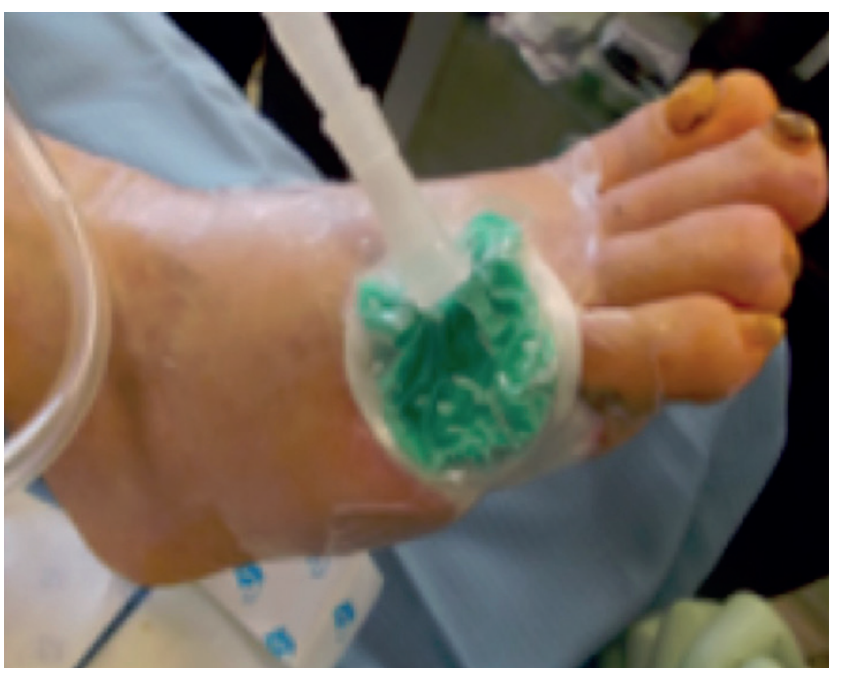

Figura 4. Applicazione di vacuum terapia. 
Wound bed preparation - Il trattamento della lesione prosegue effettuando una detersione attiva con soluzione acido-ossidante a base di acido ipocloroso, a giorni alterni, per favorire la riattivazione dei processi di guarigione. L'applicazione della soluzione è effettuata da caregiver appositamente istruito.

Decorso - L'introduzione, rispetto al trattamento già effettuato, della detersione attiva con soluzione acido-ossidante a base di acido ipocloroso si associa a una tendenza più rapida alla guarigione della lesione [Figura 5].

\section{Commenti generali sull'approccio diagnostico e} terapeutico - Il trattamento con soluzione acido-ossidante è ottimamente tollerato e ha facilitato la guarigione.

Dopo aver trattato il paziente con un intervallo medio di 1-2 giorni tra una medicazione e l'altra, abbiamo constatato gli effetti benefici della soluzione acido-ossidante laddove altri medicamenti simili, usati in precedenza, non avevano sortito risultati paragonabili. In particolare, si è potuta apprezzare la rapidità del processo di guarigione delle ferite (sia in termini di superficializzazione della lesione sia in termini di riduzione delle sue dimensioni), la tollerabilità e la facile maneg-

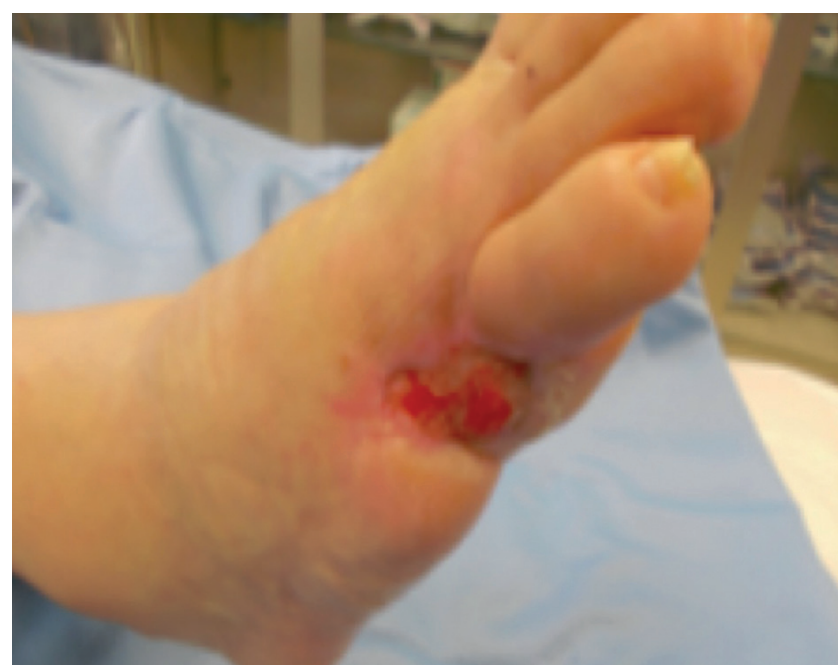

Figura 5. Aspetto della lesione dopo 5 sedute di vacuum terapia e applicazione a giorni alterni di soluzione acido-ossidante. gevolezza da parte dell'operatore, che hanno favorito la compliance del paziente.

Il trattamento con soluzione acido-ossidante è stato ottimamente tollerato e ha favorito la guarigione.

\section{Caso clinico 2 - Ulcera diabetica ingravescente dopo un trauma durante una gita al mare}

Maria Stella Aliquò

Presentazione clinica - Il paziente, diabetico, giunge all'osservazione a causa di una lesione progressivamente ingravescente, secondaria a un trauma recente

Dati anagrafici - Maschio, 57 anni.

Anamnesi patologica remota - Diabete mellito di tipo 2 con complicanze microangiopatiche (neuropatia); assenza di arteriopatia obliterante periferica.

Anamnesi patologica prossima - Il paziente riferisce di essersi procurato una ferita al I dito del piede in seguito a un urto contro uno scoglio, in occasione di una recente gita al mare. La lesione è diventata progressivamente sempre più profonda, tanto da indurre il paziente a recarsi in ospedale.

Esame obiettivo della lesione - Si evidenzia una ferita lacero-contusa infetta, a carico del I dito del piede sinistro, profonda sino alla falange ormai per buona parte persa [Figura 1].

Gestione chirurgica - Rimozione delle aree necrotiche, sutura parziale, drenaggio del materiale purulento e lavaggio interno attraverso una fistola con sodio ipoclorito allo 0,05\% [Figure 2 e 3]. Sono state eseguite mediante curette biopsie di tessuto osseo e del fondo della lesione per l'analisi microbiologica.

Wound bed preparation - Applicazione di una soluzione a base di acido ipocloroso a ogni cambio di medicazione, a giorni alterni. 


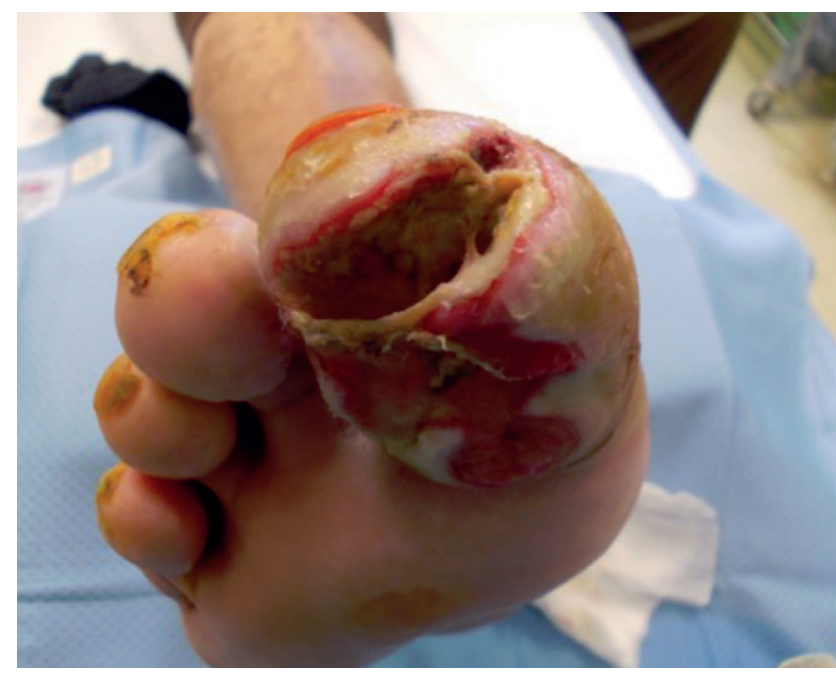

Figura 1. Aspetto della lesione alla prima osservazione.

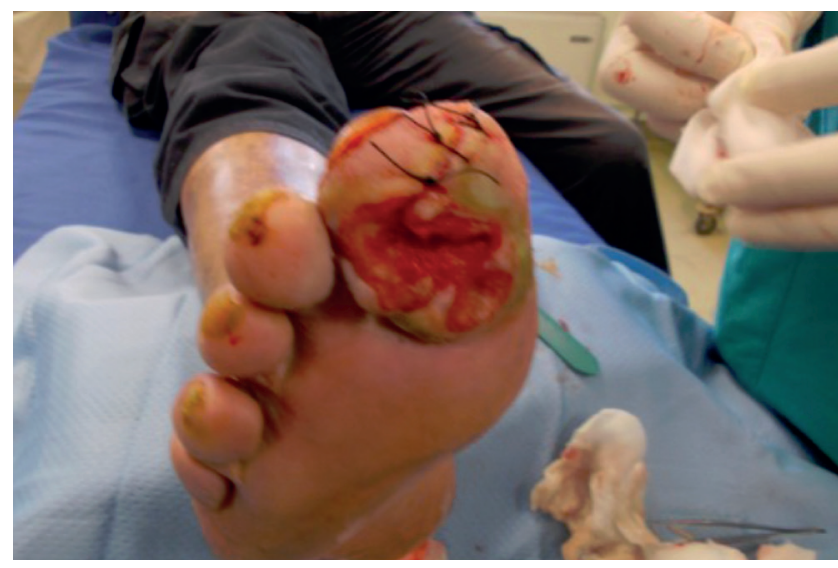

Figura 2. Rimozione della necrosi e sutura parziale.

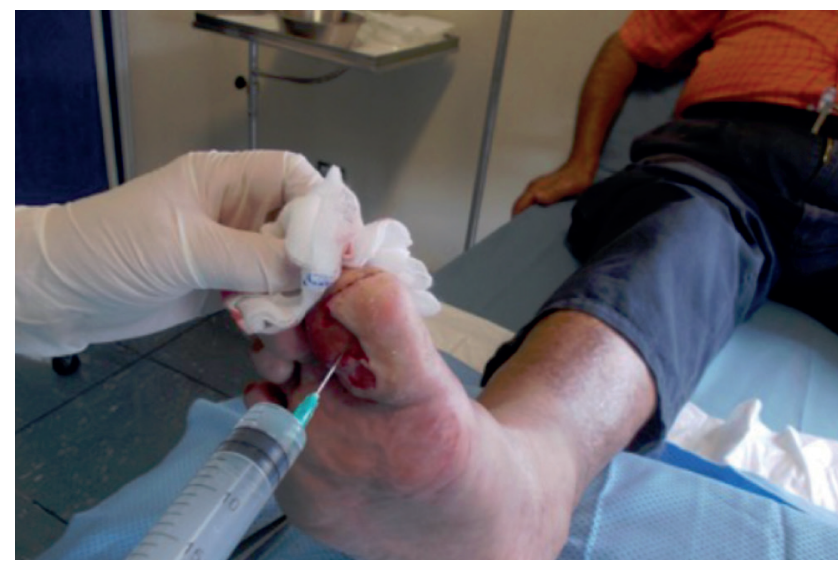

Figura 3. Lavaggio interno con sodio ipoclorito allo 0,05\% attraverso una fistola.
Decorso - La lesione è in via di guarigione e il paziente è ancora seguito presso l'ambulatorio.

\section{Commenti generali sull'approccio diagnostico} e terapeutico - Alla dimissione il paziente ha ricevuto istruzioni scritte per medicare la ferita e utilizzare la soluzione acido-ossidante. La compliance osservata è stata buona, come anche la maneggevolezza.

\section{Caso clinico 3 - Ulcera neuropatica} sovrainfetta da Pseudomonas aeruginosa in paziente diabetico Luca Monge

Presentazione clinica - Paziente affetto da diabete mellito di tipo 2 inviato da altro Ospedale alla dimissione; prima visita presso l'Ambulatorio del Piede Diabetico in data 17 ottobre 2016.

Dati anagrafici e occupazione - Maschio, 55 anni, autista.

Anamnesi patologica remota - Diabete mellito di tipo 2 noto da circa 5 anni. Ipertensione arteriosa. Fumo attivo.

Anamnesi patologica prossima - Recente ricovero in Medicina a settembre 2016 per flemmone del piede sinistro con osteomielite al $\mathrm{V}$ raggio articolazione metatarso-falangea. Lesione a partenza da ulcera neuropatica plantare della $\mathrm{V}$ testa metatarsale con sovrainfezione da Pseudomonas aeruginosa. Trattato durante il ricovero con meropenem e quindi sottoposto a bonifica chirurgica; esita un'ulcera con perdita di sostanza al dorso del piede in corrispondenza del V raggio. All'ecocolordoppler arterioso si evidenzia arteriopatia obliterante periferica diffusa senza stenosi emodinamicamente significative.

Esami ematochimici recenti: emoglobina glicata (HbA1c) 7,4\%; filtrato glomerulare stimato (eGFR) $65 \mathrm{ml} / \mathrm{min}$; $\mathrm{Hb} 15 \mathrm{~g} / \mathrm{dl}$; microalbuminuria positiva. 
Terapia in atto - Metformina, insulina con schema basal-bolus; acido acetilsalicilico, ramipril/idroclorotiazide. Trattamento antibiotico domiciliare (ADI, assistenza domiciliare integrata) con meropenem su indicazione dell'infettivologo, a cui viene abbinata una terapia orale con doxiciclina $200 \mathrm{mg} /$ die.

Esame obiettivo - Sovrappeso, neuropatia periferica documentata al monofilamento e al diapason graduato, polsi arteriosi presenti. Modesto edema declive

\section{Esame obiettivo della lesione cutanea in data 10} novembre 2016 [Figura 1]. - La lesione medicata con garza grassa e iodopovidone 10\% gel presenta uno spesso fondo di fibrina/biofilm che ricopre l'ulcera, colorito con sfumatura verdastra, sul fondo della lesione sono ben visibili i tendini, i bordi sono rilevati, edematosi. Essudato moderato.

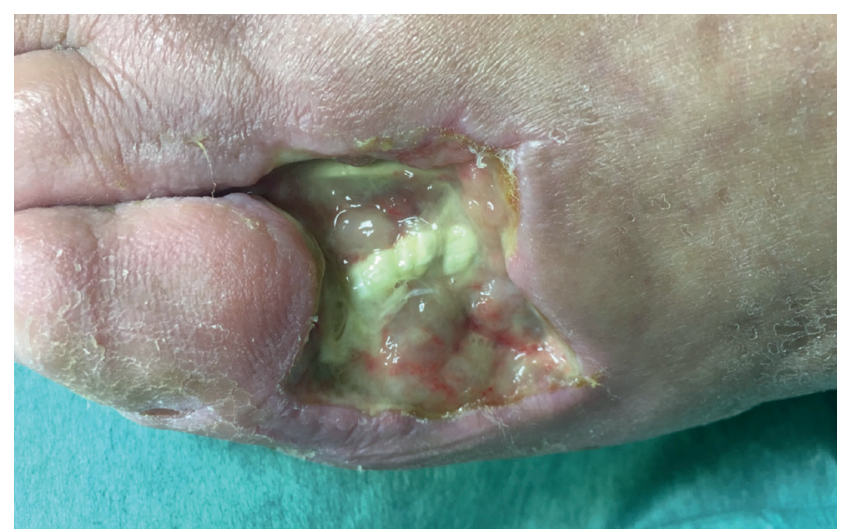

Figura 1. Situazione alla presentazione (10 novembre 2016).

Indagini di approfondimento diagnostico - Non necessarie.

Trattamento [Figura 2]. - Detersione con garza in cotone, quindi 2 spruzzi sul fondo della lesione con soluzione acido-ossidante a base di acido ipocloroso, dopo due minuti toilette con bisturi. Impacco per tre minuti con garza imbibita di sodio ipoclorito allo 0,05\%. Applicazione di idrofibra all'argento, medicazione secondaria con garza in cotone.

Prescrizione di vacuum terapia.

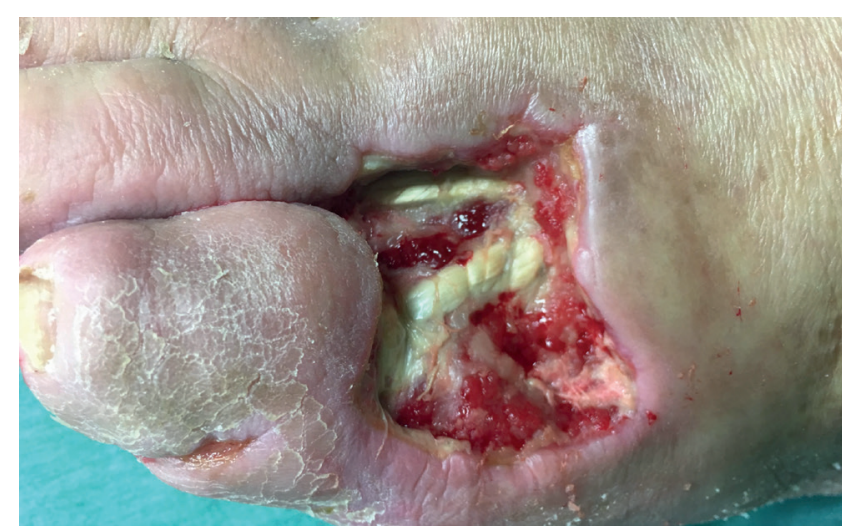

Figura 2. Situazione dopo toilette del fondo della lesione.

Nell'attesa della vacuum terapia, è prescritta una medicazione ogni tre giorni a cura del personale infermieristico dell'ASL con la seguente modalità: lavaggio con soluzione fisiologica e detersione con garza in cotone, 2 spruzzi sul fondo della lesione con soluzione acido-ossidante a base di acido ipocloroso, dopo due minuti impacco per tre minuti con garza imbibita di sodio ipoclorito allo 0,05\%. Applicazione di idrofibra all'argento, medicazione secondaria in garza di cotone.

Decorso - Dopo una settimana dalla prescrizione il paziente inizia la vacuum terapia, proseguita per 30 giorni con un cambio (spugna) ogni 4 giorni. Alla visita di controllo presso il nostro ambulatorio (12 dicembre 2016), si rileva una granulazione del tessuto con riduzione delle dimensioni della lesione; permane tendine esposto [Figura 3].

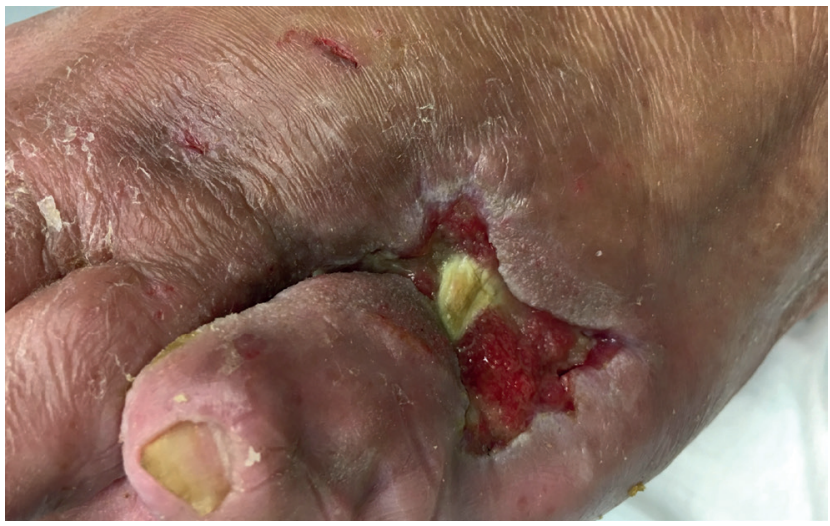

Figura 3. Situazione dopo trattamento con vacuum terapia (12 dicembre 2016). 
Trattamento - Detersione con garza in cotone, quindi 2 spruzzi sul fondo della lesione con soluzione acido-ossidante a base di acido ipocloroso, dopo due minuti si effettua una toilette del tendine. Impacco per tre minuti con garza imbibita di sodio ipoclorito allo 0,05\%. Applicazione di idrofibra all'argento, medicazione secondaria con garza in cotone. Si prescrive una medicazione ogni tre giorni a cura del personale infermieristico dell'ASL con la stessa modalità.

Decorso - Regolare; corretta esecuzione delle medicazioni. Si rileva un'ulteriore riduzione delle dimensioni della lesione che ora presenta più tessuto granuleggiante [Figura 4].

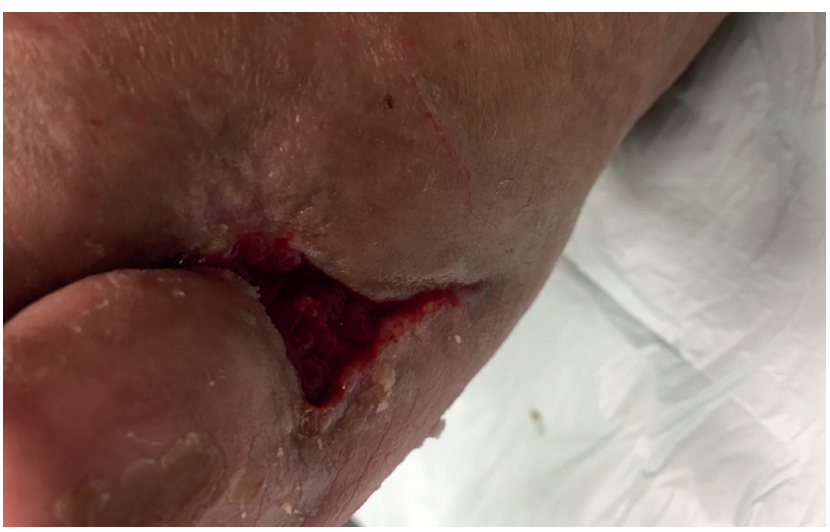

Figura 4. Situazione alla visita di controllo (12 gennaio 2017).

Trattamento - Prescritta una medicazione ogni tre giorni a cura del personale infermieristico dell'ASL con la stessa modalità in atto, sino a risoluzione dell'ulcera.

Wound bed preparation - In acuto, la sequenza della medicazione prima con soluzione acido-ossidante a base di acido ipocloroso seguita da sodio ipoclorito allo $0,05 \%$ ha permesso una disgregazione del biofilm contestualmente alla modificazione del $\mathrm{pH}$ e tale azione ha permesso una più efficace disinfezione del fondo dell'ulcera. È interessante notare come il pretrattamento per alcuni minuti con soluzione acido-ossidante a base di acido ipocloroso sembra abbia facilitato la toilette con il bisturi, rendendo il materiale fibrinoso/biofilm più facilmente rimovibile.

In cronico, la stessa medicazione con soluzione aci- do-ossidante a base di acido ipocloroso, attraverso l'elevato potenziale di ossido-riduzione e la riduzione del pH, ha permesso una riduzione della neoformazione di biofilm, una modulazione positiva delle metalloproteasi e un efficace controllo della carica microbica. Quest'ultimo è stato potenziato dalla sinergia dovuta all'uso del sodio ipoclorito allo 0,05\%, caratterizzato da ampio spettro d'azione nei confronti sia dei batteri Gram-positivi e -negativi sia di alcuni miceti, e della medicazione avanzata, che ha favorito una progressione positiva della lesione verso la granulazione e la riepitelizzazione.

Commenti generali sull'approccio diagnostico e terapeutico - Il trattamento è risultato tollerato in modo ottimale e di semplice esecuzione.

\section{Caso clinico 4 - Trattamento di ulcera neuropatica diabetica recidivante in paziente anziano attivo \\ Luca Monge}

Presentazione clinica - Paziente affetto da diabete di tipo 2 con quadro di piede neuro-vascolare, già noto al nostro ambulatorio per lesioni recidivanti.

Dati anagrafici e occupazione - Maschio, 75 anni, in pensione, ma sino allo scorso anno molto attivo come co-gestore di un campeggio al mare.

Anamnesi patologica remota - Diabete mellito di tipo 2 di lunga durata, complicato da retinopatia diabetica laser-trattata e nefropatia incipiente; ipertensione arteriosa; ipercolesterolemia. Piede neuro-vascolare in polineuropatia simmetrica distale anestetica, vasculopatia periferica trattata negli anni con PTA bilateralmente (ultima rivascolarizzazione percutanea dell'arteria femorale superficiale e dell'arteria tibiale anteriore arto destro, con buon risultato clinico, nel 2015). Nel 2012 sottoposto ad amputazione del II dito del piede destro per osteomielite. Ulcere plantari bilaterali più volte recidivanti, prevalentemente neuropatiche, trattate in fase acuta con varie tipologie di 
scarico [tutori, TCC (total contact cast)] e nelle fasi di guarigione con ortesi, calzatura con suola rigida e plantari su misura.

Anamnesi patologica prossima - A maggio 2016 ricovero in Cardiologia per coronaropatia con crisi d'angor, sottoposto a rivascolarizzazione percutanea con posizionamento di stent coronarici. Durante il ricovero non erano presenti ulcerazioni, alla ripresa della deambulazione (riabilitazione cardiologica) ricomparsa di ulcera plantare in corrispondenza della I testa metatarsale.

Terapia in atto - Metformina, empagliflozin, insulina con schema basal-bolus; doppia antiaggregazione piastrinica, atorvastatina, irbesartan, nebivololo.

\section{Esame obiettivo della lesione cutanea (3 settem-}

bre 2016) - La lesione, non adeguatamente scaricata, presenta un cercine calloso che in parte ricopre l'ulcera; il fondo della lesione è rosso con uno strato sottile di fibrina/biofilm, i bordi non sono scollati. Essudato scarso [Figura 1].

\section{Indagini di approfondimento diagnostico - Non} necessarie in presenza di un quadro vascolare compensato, programmato un tampone per esame colturale.

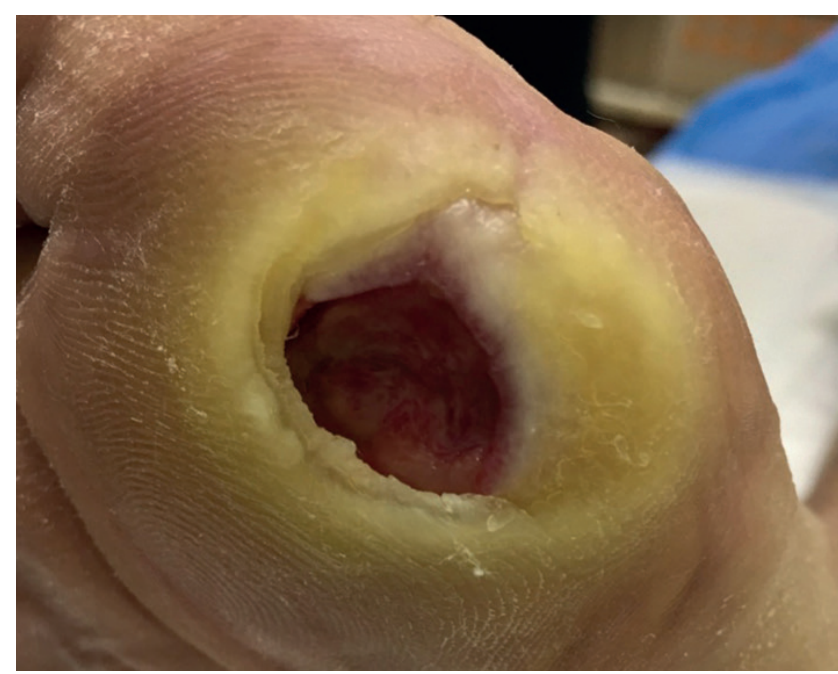

Figura 1. Aspetto della lesione alla presentazione.
Trattamento - Dopo impacco per alcuni minuti con sodio ipoclorito allo $0,05 \%$, si procede a toilette dell'ipercheratosi, quindi 2 spruzzi sul fondo della lesione con soluzione acido-ossidante a base di acido ipocloroso, dopo due minuti toilette con bisturi. Applicazione di idrofibra all'argento, medicazione secondaria. Scarico con tutore Stabil-D e soletta Modus [Figure 2 e 3 ]

Wound bed preparation - In questo caso il pretrattamento con sodio ipoclorito allo $0,05 \%$ aveva la funzione di disinfezione della lesione, mentre il trat-

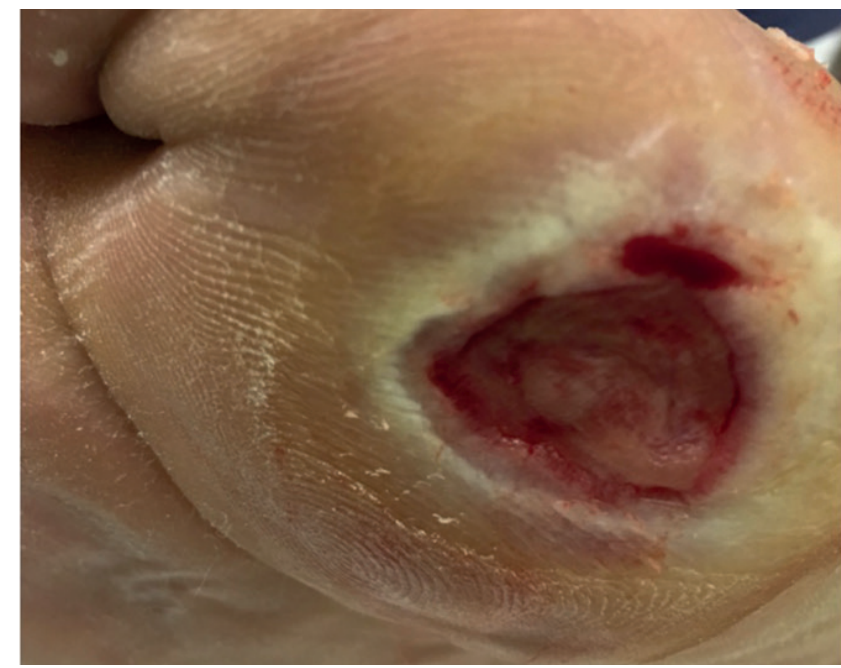

Figura 2. Toilette dell'ipercheratosi.

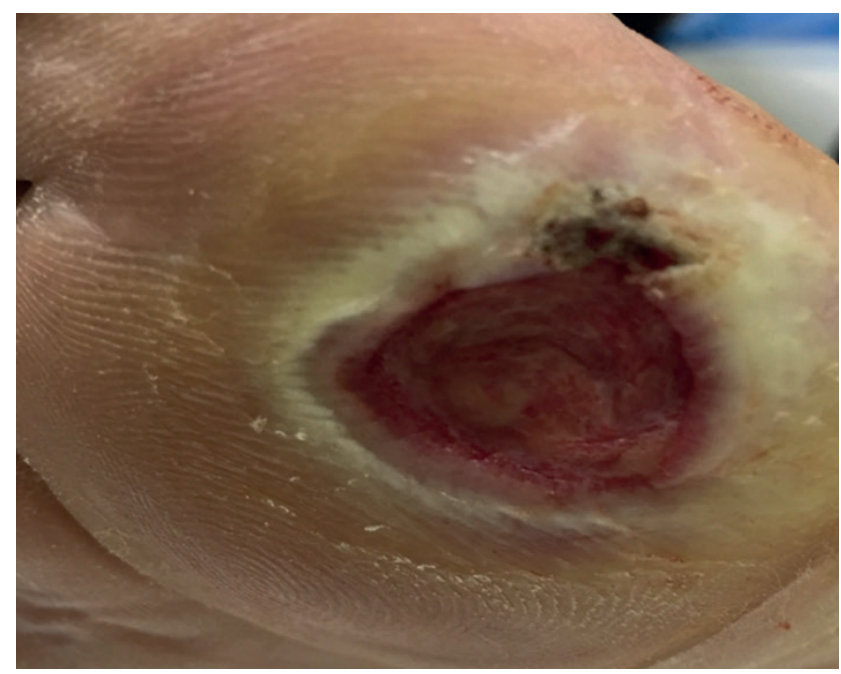

Figura 3. Aspetto della lesione dopo toilette del fondo. 
tamento del fondo della lesione con soluzione acido-ossidante a base di acido ipocloroso era finalizzato a rendere il materiale fibrinoso/biofilm più facilmente rimovibile, con riattivazione dei processi di guarigione.

La combinazione dei due trattamenti ha favorito un abbattimento del potenziale batterico della ferita attraverso l'elevato potenziale ossido-riduttivo e il basso pH dell'acido ipocloroso e l'azione diretta nei confronti dei Gram-positivi e -negativi, tipica del sodio ipoclorito.

La soluzione acido-ossidante a base di acido ipocloroso, in particolare, ha favorito la disgregazione del biofilm che riveste il fondo dell'ulcera, rendendo più facile e completa la toilette chirurgica.

\section{Commenti generali sull'approccio diagnostico e} terapeutico - Poiché la soluzione acido-ossidante a base di acido ipocloroso ha evidentemente disgregato il biofilm, potrebbe essere utile proporre in casi clinici simili la combinazione della soluzione acido-ossidante (prima) e della soluzione antisettica a base di sodio ipoclorito allo 0,05\% (dopo), con lo scopo di assicurare una più potente azione antimicrobica. È da sottolineare l'ottima tollerabilità e la semplice esecuzione del trattamento.

\section{Caso clinico 5 - Riperfusione complicata in paziente diabetico con arteriopatia obliterante periferica}

Fabio Romagnoli

Presentazione clinica - Il caso clinico descritto riguarda un paziente affetto da diabete mellito di tipo 2 complicato da arteriopatia obliterante cronica degli arti inferiori (AOP), sottoposto a numerosi interventi per ripristinare una valida perfusione periferica.

Dati anagrafici e occupazione - Maschio, 71 anni, pensionato.

Anamnesi patologica remota - Ipertensione arteriosa. Diabete mellito di tipo 2 da circa 16 anni, com- plicato da arteriopatia obliterante cronica degli arti inferiori e neuropatia diabetica.

Anamnesi patologica prossima - Sottoposto recentemente in altra struttura a rivascolarizzazione dell'arto inferiore sinistro in seguito ad accertamenti effettuati per la comparsa di una piccola lesione ulcerativa a livello della $\mathrm{V}$ testa metatarsale del piede sinistro. Giunge ai nostri ambulatori per la comparsa di necrosi alla base del $\mathrm{V}$ dito con vasto alone flogistico perilesionale. Lamenta da qualche giorno febbricola serotina e dolore al piede sinistro.

Terapia in atto - Exenatide, irbesartan, esomeprazolo, metformina, acido acetilsalicilico, lercanidipina.

Esame obiettivo della lesione cutanea - Gangrena cutanea e dei tessuti profondi alla base del $\mathrm{V}$ dito del piede sinistro con alone flogistico perilesionale $>2 \mathrm{~cm}$ e gemizio purulento da piccola lesione di continuo della cute [Figura 1].

Indagini di approfondimento diagnostico - $\mathrm{Rx}$ piede sinistro in cui si evidenzia una discontinuità della corticale del V metatarso. Ossimetria transcutanea piede sinistro: valori $35 \mathrm{mmHg}$. Ecocolordoppler arterioso degli arti inferiori: AOP in IV stadio Fontaine a sinistra. Stenosi della femorale comune e della femorale superficiale a sinistra.

Wound bed preparation - La gestione della lesione ha incluso varie fasi in funzione dei diversi momenti e procedure di rivascolarizzazione cui il paziente è stato sottoposto. Il primo intervento praticato è stata l'amputazione del V dito e della V testa metatarsale oltre all'asportazione dei tessuti necrotici, infetti e non vitali. Successivamente, per la comparsa di ischemia del IV dito [Figura 2], è stata eseguita una nuova arteriografia che ha evidenziato un'occlusione all'origine dell'arteria femorale superficiale e la presenza dell'arteria interossea quale unico vaso di gamba. Per tale motivo il paziente è stato sottoposto a by-pass femoro-popliteo e amputazione del IV raggio [Figura 3]. In seguito 


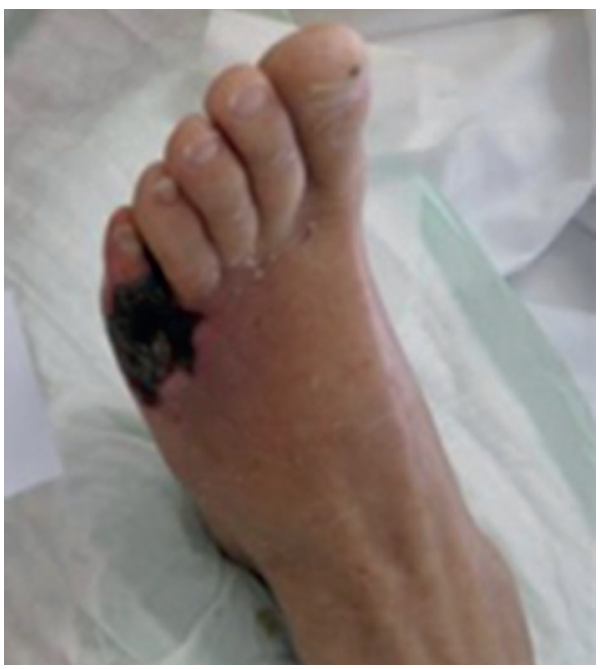

Figura 1. Aspetto della lesione alla prima visita.

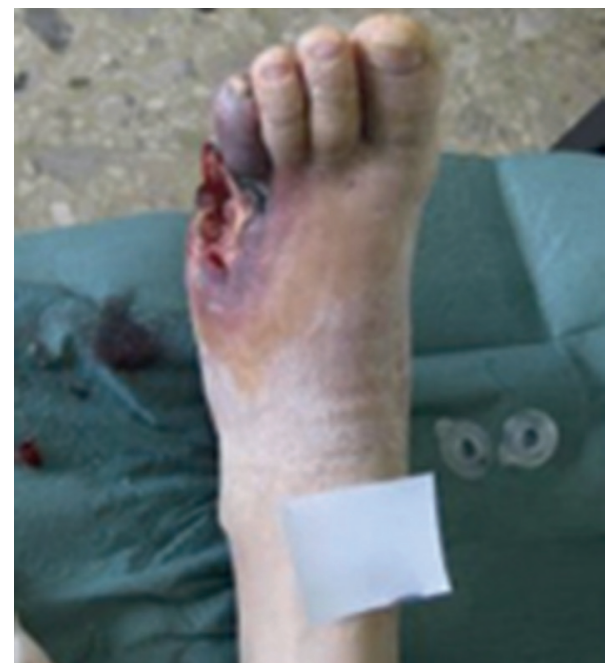

Figura 2. Evoluzione negativa dell'intervento di amputazione con comparsa di cianosi cutanea.

a questo intervento si sono resi necessari una necrosectomia dei bordi della lesione e medicazioni locali finalizzate a ottenere una valida detersione del fondo. Successivamente è stato applicato un innesto con un sostituto dermico artificiale. L'evoluzione favorevole della lesione si è arrestata dopo circa 3 settimane con la comparsa di un fondo fibrinoso e necrosi dei bordi [Figura 4]. Un'ulteriore arteriografia ha evidenziato un'occlusione a livello del confezionamento del by-pass. Il paziente è stato quindi sottoposto a trattamento delle occlusioni

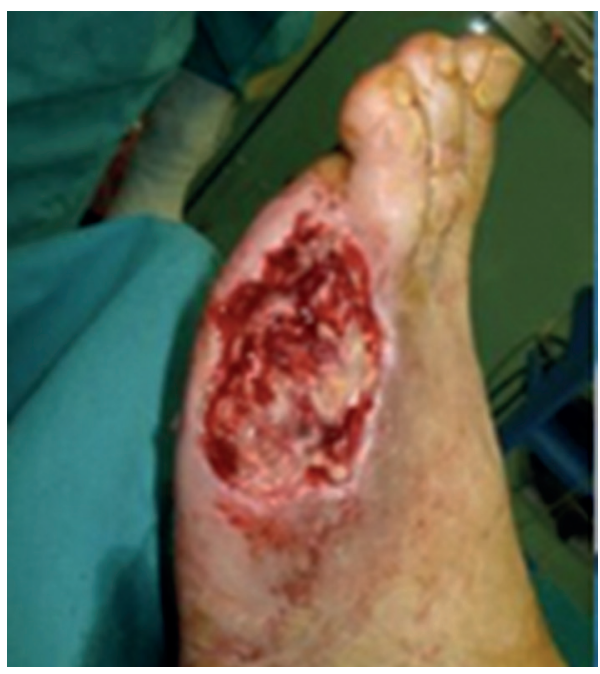

Figura 3. Quadro clinico dopo intervento di amputazione del IV dito.

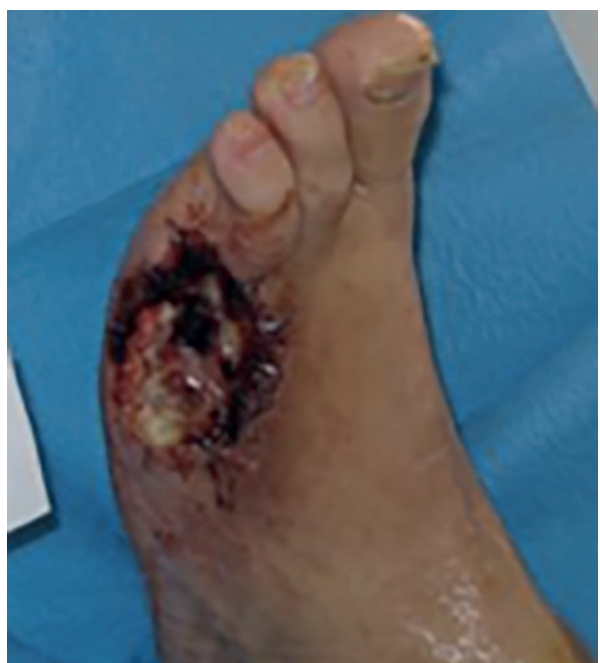

Figura 4. Blocco del processo di guarigione con comparsa di fondo sanioso e bordi necrotici.

mediante angioplastica percutanea (PTA). Il successivo approccio terapeutico della lesione con soluzione acido-ossidante a base di acido ipocloroso e garza vasellinata è stato finalizzato a ottenere un fondo ben deterso [Figura 5], in grado di accogliere uno skin graft. I controlli successivi [Figura 6] hanno mostrato un'evoluzione favorevole dell'ulcera.

Decorso - Il decorso del caso clinico presentato ha mostrato come la vascolarizzazione periferica sia una condizione indispensabile nel trattamento delle lesio- 


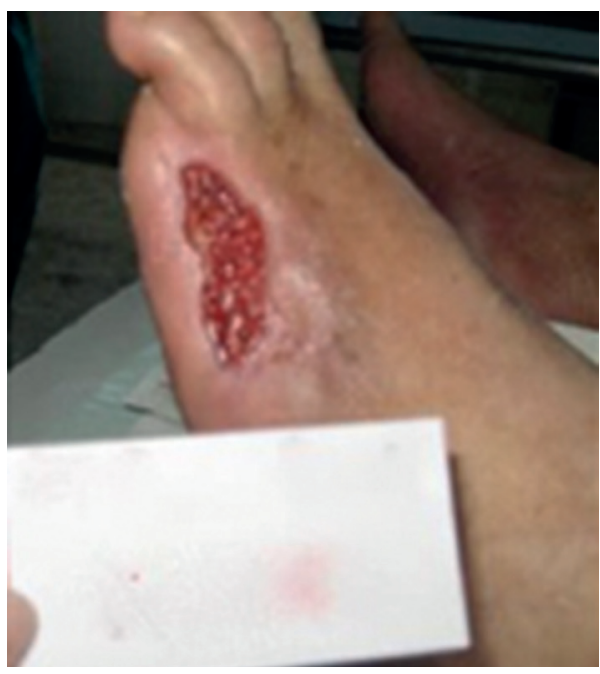

Figura 5. Quadro clinico post-innesto di sostituto dermico artificiale.

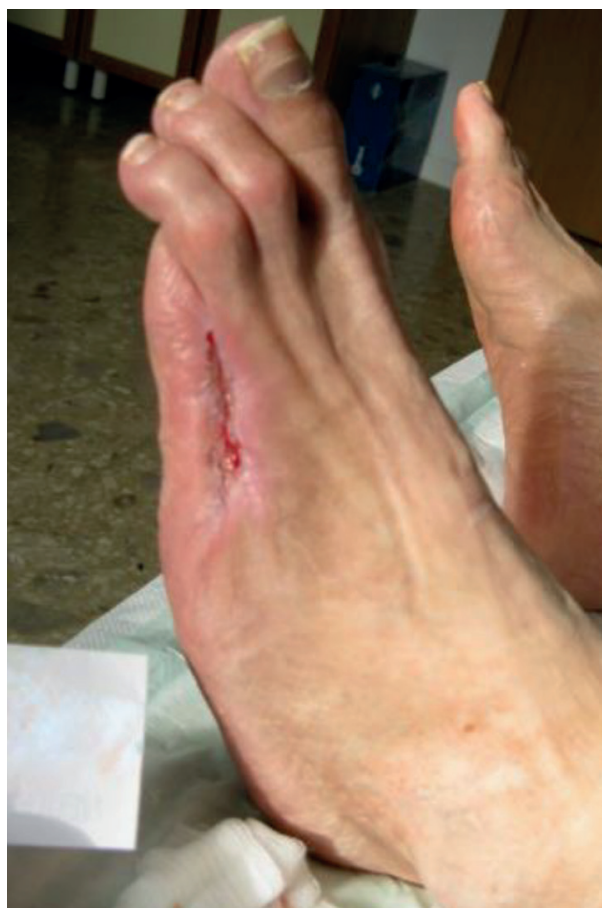

Figura 6. Quadro post-innesto cutaneo.

ni cutanee, tra cui il piede diabetico: l'evoluzione positiva infatti è stata ottenuta grazie al conseguimento di una sufficiente perfusione.

\section{Commenti generali sull'approccio diagnostico} e terapeutico - Il trattamento delle lesioni del piede diabetico ischemico è particolarmente comples- sa e impegnativa. Le recidive sono estremamente frequenti: $25-30 \%$ entro un anno, $50 \%$ in 3 anni e il $75 \%$ in 7 anni. In questo caso l'arteriopatia era caratterizzata da un interessamento sia dei grossi vasi (femorale superficiale) sia dei vasi sottogenicolati che ha reso necessari interventi di rivascolarizzazione sia di tipo chirurgico sia di radiologia interventistica. Il trattamento della lesione ha quindi richiesto una valutazione sempre molto accurata correlando la scarsa evoluzione verso la guarigione o il suo peggioramento alla situazione circolatoria periferica, risultata sempre molto instabile. Un aspetto da tenere presente è che non sempre sono utili le medicazioni avanzate. È fondamentale ottenere una buona vascolarizzazione periferica e mettere in scarico la lesione con l'uso di tutori (a suola rigida o a barchetta) per evitare il continuo insulto sulla lesione.

\section{Caso clinico 6 - Ulcera cronica post-traumatica in paziente con artrite reumatoide \\ Rolando Tasinato}

Presentazione clinica - La paziente è giunta alla nostra osservazione a distanza di circa 2 settimane da un trauma, avvenuto urtando accidentalmente la superficie laterale esterna della gamba destra contro un oggetto contundente.

Dati anagrafici - Femmina, 62 anni.

Anamnesi patologica remota - Paziente affetta da artrite reumatoide da anni, in trattamento con corticosteroidi e immunosoppressori.

Anamnesi patologica prossima - Dopo il trauma alla gamba la paziente ha sviluppato un'ecchimosi della superficie contusa, la cute in corrispondenza del trauma è andata incontro a necrosi nell'arco di circa una settimana con comparsa di intensa flogosi perilesionale e limitazione funzionale dell'arto alla deambulazione, già di per sé fortemente limitata dal- 


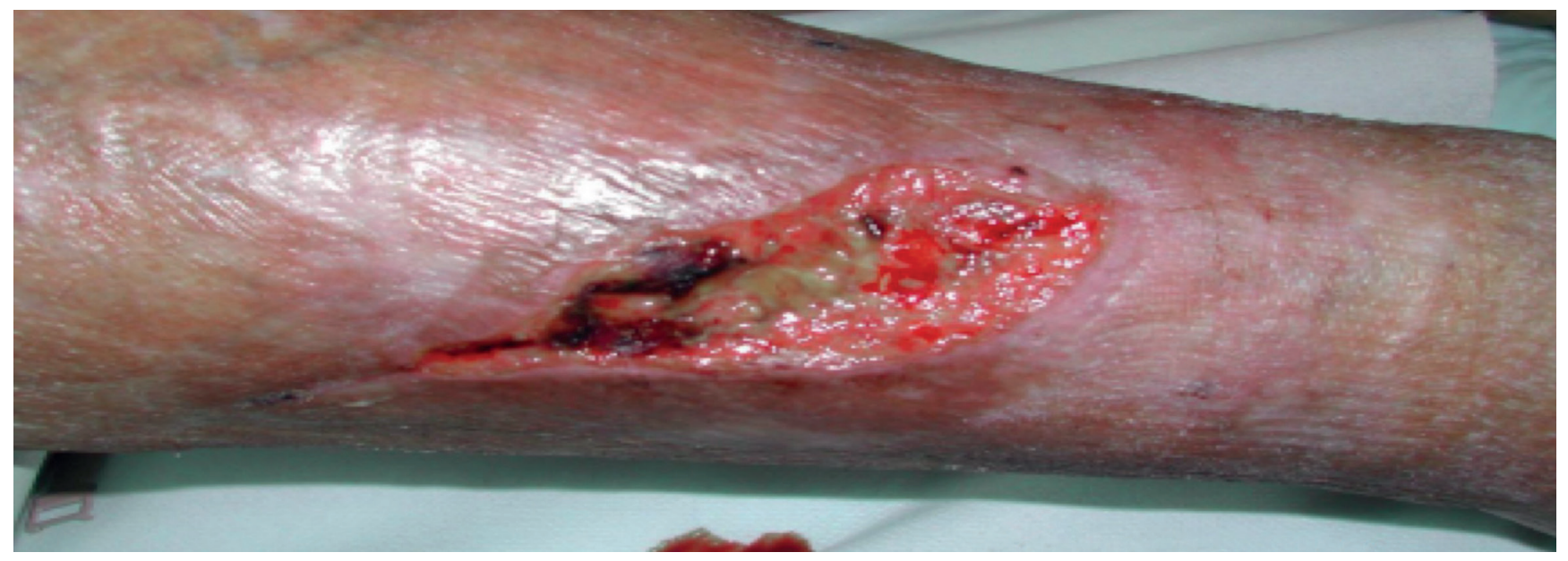

Figura 1. Lesione post-traumatica in paziente con artrite reumatoide in trattamento immunosoppressivo e corticosteroideo. Aspetto della lesione alla prima visita in ambulatorio (26 maggio 2016).

la patologia osteoarticolare preesistente. La paziente è stata trattata inizialmente dal medico di famiglia con medicazioni topiche a base di sulfadiazina argentica e terapia antibiotica per os con levofloxacina $500 \mathrm{mg}$, due volte al giorno per 8 giorni, e in seguito inviata al nostro ambulatorio vulnologico.

Esame obiettivo della lesione - Si evidenzia un'ulcera cronica di natura post-traumatica [Figura 1].

\section{Indagini di approfondimento diagnostico - Non} necessarie.
Trattamento - Nel corso della prima visita abbiamo proceduto al curettage chirurgico in anestesia locale topica con lidocaina 10\% (nebulizzazione diretta della lesione). Nei giorni successivi la lesione è stata sottoposta a debridement enzimatico con collagenasi, antisepsi quotidiana con sodio ipoclorito allo 0,05\% e medicazione assorbente con alginato.

Decorso - Successivamente al debridement, abbiamo osservato una riduzione della flogosi perilesionale associata a un miglioramento netto della sintomatologia dolorosa.

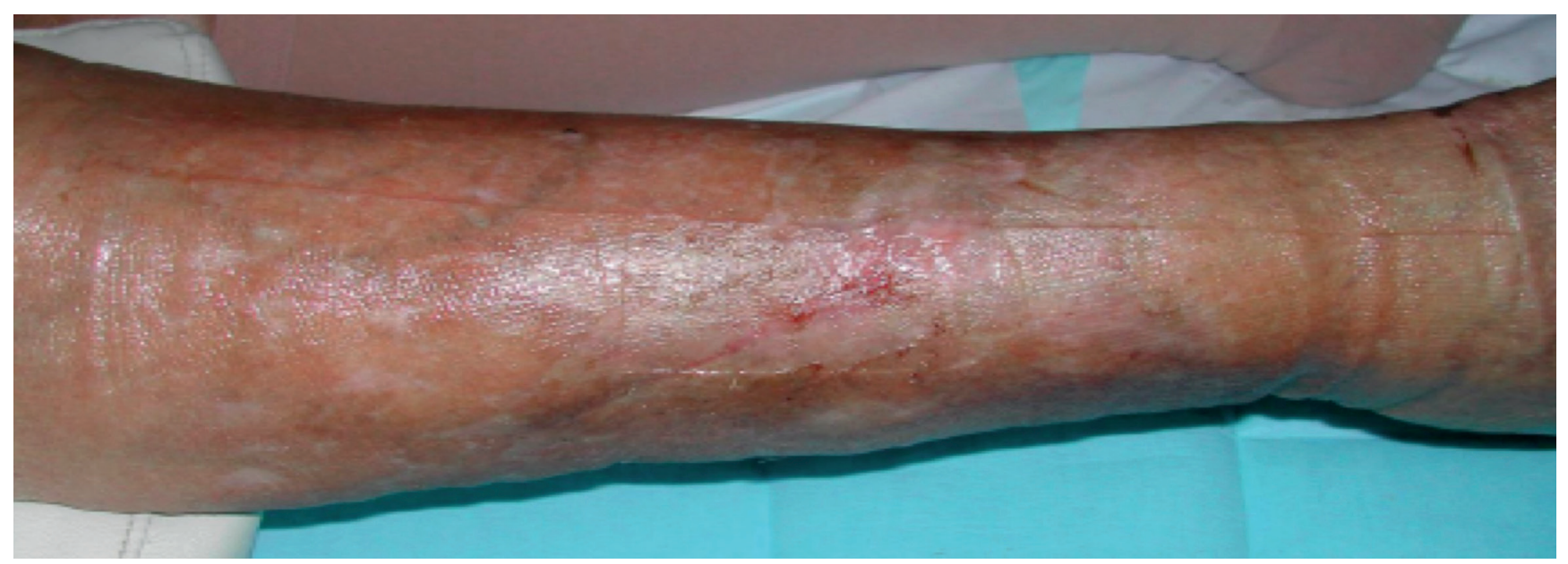

Figura 2. Aspetto della lesione dopo 6 settimane dall'inizio del trattamento secondo il protocollo TIME (Tissue management, Inflammation and infection control, Moisture balance, Epithelial (edge) advancement) (8 luglio 2016). 
Wound bed preparation - Una volta cessata la fase di secrezione e ultimato il debridement (18 giorni dopo la prima visita), siamo passati a effettuare una detersione attiva con una soluzione acido-ossidante a base di acido ipocloroso. Tale trattamento ha consentito un'ulteriore detersione del fondo dell'ulcera, favorendo la successiva riepitelizzazione che si è conclusa a distanza di 6 settimane dalla prima osservazione presso il nostro ambulatorio [Figura 2].

Decorso - Nella fase di trattamento della lesione non è stata sospesa la terapia con immunosoppressori, mentre nelle 8 settimane successive al trauma è stato ridotto il dosaggio della terapia corticosteroidea del 50\% rispetto al dosaggio abituale in cronico.

Commenti generali sull'approccio diagnostico e terapeutico - Attualmente la paziente ha ripreso la terapia immunosoppressiva e steroidea abituale, e non presenta limitazioni funzionali dell'arto correlate al trauma o recidiva della lesione ulcerativa.

\section{Caso clinico 7 - Ulcera flebostatica in paziente con insufficienza venosa Rolando Tasinato}

Presentazione clinica - Il paziente giunge all'osservazione per ulcera flebostatica sul dorso del piede destro da pregressa trombosi venosa profonda (TVP) con insufficienza venosa cronica dell'arto inferiore destro in classe CEAP C6EsApPr,o.

Dati anagrafici - Maschio, 57 anni.

Anamnesi patologica remota - Il paziente presenta insufficienza venosa cronica da circa 20 anni, nel corso dei quali è stato sottoposto a tre interventi chirurgici a carico del circolo venoso superficiale. Sei anni prima aveva avuto una trombosi venosa profonda dell'arto inferiore destro, conseguente a immobilizzazione prolungata da apparecchio gessato. Successiva- mente, nel 2013, aveva sviluppato una piccola ulcera flebostatica in sede perimalleolare, trattata con bendaggio elastocompressivo e 4 sedute di trattamento con scleromousse (sodio tetradecilsolfato 1\%) terapia iniettiva condotta su alcune varici reticolari perimalleolari sempre del piede destro.

Anamnesi patologica prossima - Giunge alla nostra osservazione con una lesione atipica del dorso del piede, che a una valutazione ecodoppler viene imputata al reflusso breve settoriale proveniente da una vena perforante perimalleolare che alimenta una varice particolarmente ectasica sul dorso del piede. Eczema varicoso e ipodermite concomitanti, anch'essi correlati allo stato di insufficienza venosa cronica e presenti già in fase pre-ulcerativa.

Esame obiettivo della lesione - Alla prima visita presso il nostro ambulatorio, la lesione appare relativamente ben detersa, con diametro di circa $2 \mathrm{~cm}$, non sanguinante e non particolarmente secernente.

Trattamento - Viene inizialmente avviato un trattamento che prevede antisepsi con sodio ipoclorito allo 0,05\% e bendaggio elastocompressivo.

Wound bed preparation - Una volta ottenuto lo sbrigliamento (2 sole medicazioni in quanto la lesione già all'esordio si presentava con fondo discretamente pulito), si decide di effettuare la detersione del fondo dell'ulcera con soluzione acido-ossidante di acido ipocloroso seguita sempre da medicazione protettiva (garza grassa e bendaggio elastocompressivo multistrato).

Decorso - Le Figure 1 e $\mathbf{2}$ evidenziano la lesione a distanza di 16 giorni una dall'altra: durante questo periodo il paziente è sottoposto complessivamente a 6 medicazioni dell'ulcera. Accanto alla detersione e al bendaggio elastocompressivo, il paziente è stato invitato a deambulare attivamente per 30 minuti, due volte al giorno, fino alla riepitelizzazione della lesione. 


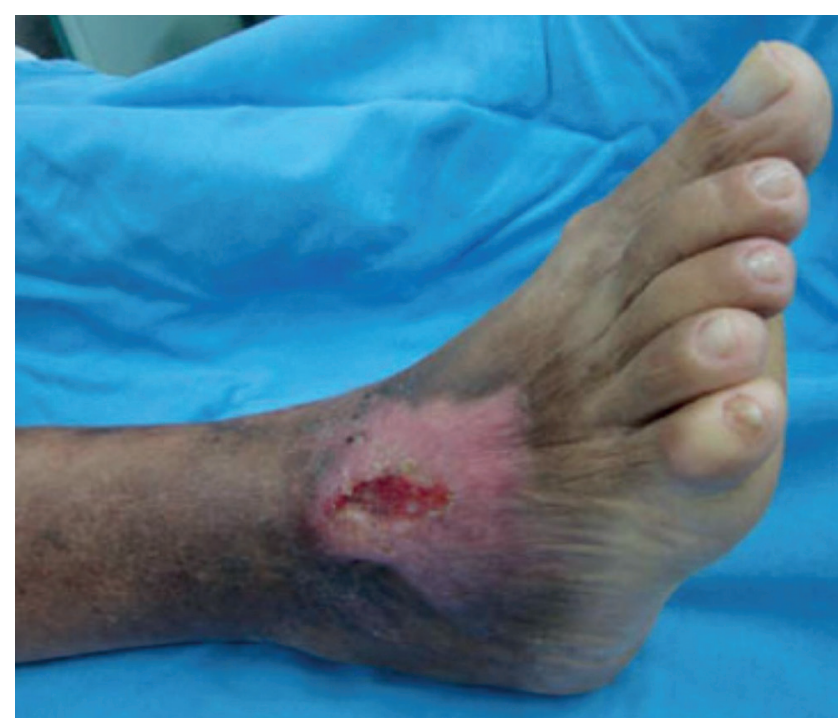

Figura 1. Ulcera flebostatica in paziente con insufficienza venosa e pregressa trombosi venosa profonda.

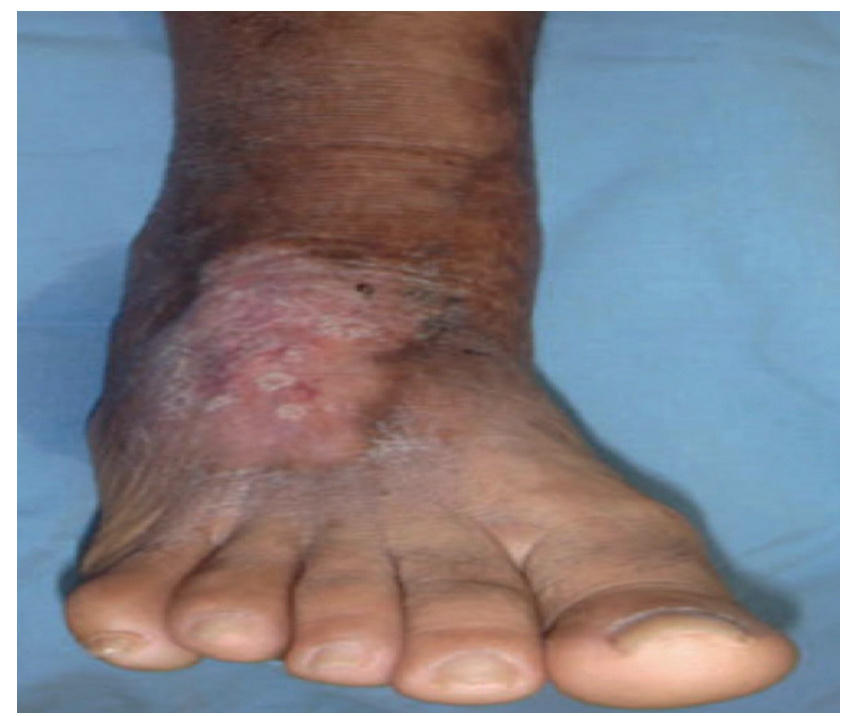

Figura 2. Risultato dopo 16 giorni di trattamento con debridement, detersione con sodio ipoclorito allo 0,05\% e soluzione acido-ossidante con acido ipocloroso e successivo bendaggio elastocompressivo.

\section{Commenti generali sull'approccio diagnosti-}

co e terapeutico - Il trattamento con soluzione acido-ossidante ha consentito una detersione attiva dell'ulcera e ne ha favorito la riepitelizzazione. Il successivo trattamento profilattico per evitare la recidiva ha previsto l'uso diurno di un gambaletto elastico della 2a classe di compressione KKL2.

\section{Protocollo per la preparazione del letto della ferita}

La serie di casi clinici riportati testimonia da una parte la necessità di un management della lesione ulcerativa che prenda in considerazione le caratteristiche del contesto clinico in cui si sviluppa la lesione e le condizioni generali (stato di salute, condizioni socio-economiche) del paziente e dall'altra il significativo aiuto ai fini della guarigione di una preparazione ottimale del letto della ferita.

In questi anni un punto di svolta nell'ambito della vulnologia è stata la pubblicazione del position paper Wound Bed Preparation in Practice della European Wound Management Association, che ha proposto il protocollo TIME (Tissue management, Inflammation and infection control, Moisture balance, Epithelial (edge) advancement), un sistema per la gestione delle procedure da attuare volto a indicare quali elementi occorra correggere nella preparazione del letto della ferita [26]. Vediamo nel dettaglio i punti salienti del protocollo.

- Le cellule non vitali impediscono la guarigione: è pertanto necessario ripristinare il fondo della ferita e le funzioni della matrice extracellulare attraverso il debridement episodico o continuo.

- La presenza di una elevata carica batterica e di infiammazione provoca un incremento delle citochine infiammatorie e dell'attività proteasica, con riduzione dell'attività dei fattori di crescita. Bisogna quindi agire contro il bioburden batterico e ridurre la flogosi attraverso l'uso topico di soluzioni antisettiche e, se necessario, di antibiotici topici e sistemici ricordando sempre l'importanza del problema delle resistenze batteriche.

- Altro aspetto da non trascurare è il mantenimento di un corretto livello di idratazione: la disidratazione cutanea rallenta infatti la migrazione delle cellule epiteliali, mentre un eccesso di liquidi causa la macerazione dei margini della ferita. In questi casi occorre applicare medicazioni che mantengano il corretto grado di umidificazione tissutale e rimuovere i liquidi utilizzando la compressione, la pressione negativa o altre metodiche. 
- Infine vanno valutati i margini dell'epidermide: i cheratinociti che non migrano e le cellule della ferita che non rispondono agli stimoli impediscono la rimarginazione dei tessuti. Occorre pertanto assicurare la migrazione dei cheratinociti e la risposta delle cellule della ferita prendendo in considerazione terapie correttive come debridement, trapianti cutanei, prodotti biologici o terapie aggiuntive.

È utile ribadire che la preparazione del letto di una ferita non coincide con la sola rimozione del tessuto necrotico (debridement), ma deve tenere in considerazione anche la presenza di essudato e infezione e la loro eliminazione.

\section{Soluzione acido-ossidante a base di acido ipocloroso: sinergia di intervento}

Il secondo caso descritto dal dr. Luca Monge evidenzia i vantaggi di un uso sequenziale della soluzione acido-ossidante a base di acido ipocloroso e delle soluzioni di sodio ipoclorito, per sfruttare in modo ottimale le peculiarità dei due prodotti.

L'utilizzo di una soluzione con caratteristiche chimico-fisiche particolari, come $\mathrm{pH}$ acido, elevato potenziale di ossido-riduzione e contenuto di acido ipocloroso, permette di modulare il microambiente della ferita. L'effetto di riduzione del $\mathrm{pH}$ della lesione ha, infatti, come conseguenza anche la modulazione dell'attività delle metalloproteasi, fenomeno che contribuisce a facilitare la guarigione della ferita [14].

Il $\mathrm{pH}$ acido e la presenza dell'acido ipocloroso, oltre all'elevato potenziale di ossidoriduzione, influiscono sulla riduzione della carica batterica creando un ambiente sfavorevole allo sviluppo dei batteri (biofilm); inoltre regolano il processo di ossidazione dovuto alle attività cellulari e ai batteri. L'azione di acidificazione nell'ambiente della ferita può aumentare la percentuale di ossigeno grazie alla dissociazione e riduzione dell'istotossicità dei prodotti finali del metabolismo batterico [14].

La soluzione acido-ossidante a base di acido ipocloroso, inoltre, disgrega la struttura di protezione prodotta dai batteri che li rende resistenti ad altri trattamenti e consente di portare i germi in forma planctonica e quindi più sensibili a ulteriori interventi terapeutici con antisettici o antibiotici [27]. I microrganismi all'interno del biofilm hanno una replicazione più lenta $\mathrm{e}$ molti antibiotici non riescono ad agire efficacemente. In presenza di segni clinici di infezione è indicato anche l'utilizzo della soluzione antisettica a base di sodio ipoclorito allo $0,05 \%$. È stato documentato un effetto batteriostatico e battericida sovrapponibile tra le soluzioni perossidanti e l'ipoclorito di sodio [28]. Come proposto dal dr. Monge, ai fini di una detersione, pulizia e disinfezione ottimali del letto della ferita infetta, l'azione più efficace è dunque l'utilizzo sequenziale di entrambe le soluzioni, cioè la soluzione a base di acido ipocloroso a coprire il fondo della ferita, che va lasciata agire per 2 minuti in modo da poter esplicare la sua azione di detersione attiva e di alterazione del glicocalice del biofilm, seguita dal sodio ipoclorito allo $0,05 \%$ dotato di attività antisettica ad ampio spettro.

Entrambi i prodotti non danneggiano il tessuto di granulazione; inoltre il $\mathrm{pH}$ acido della soluzione acido-ossidante tende a favorire l'ossigenazione dei tessuti e impedisce ai prodotti azotati, derivati dal catabolismo dei batteri e dei tessuti stessi, di penetrare nelle cellule sane circostanti la ferita rendendole sofferenti [29].

In un modello di lesione cutanea profonda, in presenza di soluzione acido-ossidante a base di acido ipocloroso si è osservata una significativa riduzione del richiamo di neutrofili nel sito infiammatorio della lesione a vantaggio del processo di guarigione e senza effetti sulla formazione del tessuto di granulazione [29]. Parallelamente, al fine di escludere una possibile azione irritante sulla cute, ne è stata valutata la tollerabilità in vivo. Il prodotto, sia dopo applicazione singola sia dopo applicazione ripetuta per 4 settimane, non ha determinato alcun effetto irritativo sulla cute del coniglio né in termini di risposta eritematosa né di formazione di edema, dimostrando quindi un'ottima tollerabilità e rivelandosi adatto a un utilizzo anche in condizioni di ferite profonde [29].

La soluzione acido-ossidante a base di acido ipocloroso in formato spray viene spruzzata sul letto di fe- 
rita, facilitando l'azione meccanica di rimozione dei detriti dalla lesione. Il tempo di contatto è di circa 2 minuti. Nel caso di uno sbrigliamento chirurgico, ne viene consigliato l'utilizzo prima e dopo la rimozione del tessuto devitalizzato. Se ne sconsiglia invece l'uso simultaneo con prodotti enzimatici per il debridement, dei quali è necessario considerare le caratteristiche: alcune collagenasi esplicano infatti la loro attività a un $\mathrm{pH}$ compreso tra 6 e 8. In generale, l'utilizzo della soluzione acido-ossidante non ha comunque dimostrato nella pratica clinica interazioni con la medicazione primaria.

Nell'approccio alla lesione ci si dovrebbe sempre chiedere qual è l'obiettivo che si desidera ottenere rispetto al trattamento e qual è l'obiettivo rispetto a una visione olistica del paziente. Una documentazione strutturata sulla storia del paziente e sui parametri clinici della lesione rappresenta il punto di partenza della best practice e della gestione multidisciplinare del piano di cura. La valutazione della lesione, aspetto fondamentale del percorso di trattamento, deve considerare le dimensioni della ferita, il tessuto presente, la quantità di essudato, i segni clinici di colonizzazione critica/infezione, l'arresto e/o la progressione dei bordi, la presenza di dolore.

\section{La valutazione della ferita: agire sulle cause}

Nell'ambito del trattamento globale delle ferite resta fondamentale l'agire sulle cause sottostanti $[9,30]$. È estremamente importante assicurarsi che sia presente un adeguato apporto ematico, grazie al quale è possibile un più rapido processo di cicatrizzazione [30]. Ł̀ quindi essenziale diagnosticare un'eventuale patologia vascolare all'inizio del trattamento, in particolare nei pazienti affetti da ulcere degli arti inferiori o da disordini metabolici quali il diabete [30]. In assenza di un'adeguata perfusione, si avrà una carenza di apporto di nutrienti e di ossigeno, ambedue necessari per supportare la forte richiesta metabolica associata al processo di cicatrizzazione. Inoltre, anche l'afflusso delle componenti cellulari del sistema immunitario (leucociti e macrofagi), fondamentali per il processo di cicatrizzazione, risulta ridotto in caso di perfusione alterata. La presenza di un'insufficienza venosa, infine, influenza negativamente il processo di cicatrizzazione, impedendo la rimozione spontanea dei detriti dal letto dell'ulcera [31].

Un punto a cui deve essere prestata grande attenzione riguarda il fatto che il contesto clinico in cui si sviluppa la ferita risente oltre che di fattori fisici, come appunto le patologie sottostanti e le comorbilità, anche di aspetti psicologici e del setting sociale [11] La complessità del quadro clinico si riverbera inevitabilmente sulla qualità della vita sia del paziente sia dei familiari, causando spesso disagi nelle quotidianità [32,33].

Una lesione che non guarisce rappresenta una fonte di preoccupazione non indifferente per un paziente, e gioca un ruolo fondamentale nel valutare negativamente la propria qualità di vita.

Diversi fattori psicosociali, quali ansia, depressione, isolamento sociale, condizioni disagiate, privazione del sonno e dolore, conducono questi pazienti verso una spirale negativa che li induce a ridurre la vita sociale, aumentare l'isolamento e di conseguenza a perdere la fiducia in sé stessi e nelle proprie capacità arrivando anche alla sfiducia nel trattamento e al suo abbandono. Questi fattori sono particolarmente rilevanti nei casi di ulcere venose refrattarie in una popolazione anziana. La conoscenza dei vissuti e delle problematiche del vivere con un'ulcera, del percorso intrapreso tra professionisti e servizi, delle speranze e aspettative che le persone nutrono nei confronti della guarigione dell'ulcera è fondamentale per comprendere in profondità cosa significhi vivere con questa problematica.

Risulta quindi fondamentale il coinvolgimento nel percorso di cura anche di figure professionali appropriate come gli psicologi e assistenti sociali [11].

\section{Conclusioni}

La cura dell'ulcera "difficile" è un processo articolato e complesso, in cui è necessaria una visione globale 
e multispecialistica del quadro clinico. La scelta degli interventi medici più idonei a facilitare la guarigione della lesione, tra cui in primis la corretta preparazione del letto della ferita, rappresenta il cuore dell'attività dell'esperto in vulnologia. Tuttavia individuare le esigenze e le preoccupazioni del paziente, ricono-

\section{Disclosures}

Gli autori dichiarano di non avere conflitto di interessi inerente all'articolo.

\section{Bibliografia}

1. Markova A, Mostow EN. US skin disease assessment: ulcer and wound care. Dermatol Clin 2012;30:107-11,ix

2. S3-Guideline on Venous Leg Ulcer Developed by the Guideline Subcommittee 'Diagnostics and Treatment of Venous Leg Ulcers' of the European Dermatology Forum JEADV 2016;30:1843-75. https:// www.deepdyve.com/lp/wiley/evidence-based-s3-guidelines-fordiagnostics-and-treatment-of-venous-XXMx1CRDq9

3. Cook JJ, Simonson DC. Epidemiology and health care cost of diabetic foot problems. In: Veves A, Giurini JM, LoGerfo FW (eds). The Diabetic Foot: Medical and Surgical Management, Contemporary Diabetes, 1. doi 10.1007/978-1-61779-791-0_2

4. Guest JF, Ayoub N, McIlwraith T et al. Health economic burden that wounds impose on the National Health Service in the UK. BMJ Open 2015;5:e009283. doi 10.1136/ bmjopen-2015-009283

5. Guest JF, Ayoub N, McIlwraith T et al. Health economic burden that different wound types impose on the UK's National Health Service. Int Wound J 2017 Apr;14(2):322-30. doi 10.1111/iwj.12603

6. Mustoe TA, O'Shaughnessy K, Kloeters O. Chronic wound pathogenesis and current treatment strategies: a unifying hypothesis. J Plast Reconstr Surg 2006;117(Suppl 7):35S-41S

7. White-Chu EF, Reddy M. Wound care in short-term rehabilitation facilities and long-term care: special needs for a special population. Skinmed 2012 Mar-Apr;10(2):75-81

8. Takahashi PY, Kiemele LJ, Jones JP Jr. Wound care for elderly patients: advances and clinical applications for practicing physicians. Mayo Clin Proc 2004 Feb;79(2):260-7

9. Falanga V. Classifications for wound bed preparation and stimulation of chronic wounds. Wound Repair Regen 2000;8:347-52

10. Flanagan M. Wound Healing and Skin Integrity: Principles and Practice. Wiley-Blackwell 2013

11. European Wound Management Association (EWMA). Documento di Posizionamento. Ferite di difficile guarigione: un approccio olistico. MEP Ldt 2008

12. Caley MP, Martins VLC, O'Toole EA. Metalloproteases and wound healing. Advances in Wound Care 2015 Apr;4(4):225-34

13. Greener B, Hughes AA, Bannister NP, Douglass J. Proteases and pH in chronic wounds. J Wound Care 2005;14(2):59-61 scere e discutere i possibili ostacoli alla guarigione, fornire assistenza ricorrendo dove necessario ad altri specialisti e occuparsi del paziente nel suo insieme sono altri aspetti fondamentali da tenere sempre presenti per il miglioramento dell'esperienza di malattia di persone fragili in cerca di aiuto.

14. Percival SL, McCarty S, Hunt JA, Woods EJ. The effect of pH on wound healing, biofilms, and antimicrobial efficacy. Wound Repair Regen 2014;22:174-86

15. Fluhr JW, Kao J, Jain M et al. Generation of free fatty acids from phospholipids regulates stratum corneum acidification and integrity. J Invest Dermatol 2001;117:44-51

16. The dermatologist. Journal of Dermatology, Venerology and related areas. The milieu of chronic wounds. Investigations as part of a modern wound therapy. Springer-Verlag 2003. http:// www.thblack.com/links/RSD/pH_chronic_wounds.pdf

17. Charbonneau NL, Carlson EJ, Tufa $\mathrm{S}$ et al. In vivo studies of mutant fibrillin-1 microfibrils. J Biol Chem 2010;285(32):24943-55

18. Schneider LA, Korber A, Grabbe S, Dissemond J. Influence of pH on wound-healing: a new perspective for wound-therapy? Arch Dermatol Res 2007;298 (9):413-20

19. Bowler PG, Duerden BI, Armstrong DG. Wound microbiology and associated approaches to wound management. Clin Microbiol Rev 2001;14(2):244-69

20. Gardner SE, Frantz RA, Doebbeling BN. The validity of the clinical signs and symptoms used to identify localized chronic wound infection. Wound Repair Regen 2001;9(3):178-86

21. Kostakioti M, Hadjifrangiskou M, Hultgren SJ. Bacterial biofilms: development, dispersal, and therapeutic strategies in the dawn of the postantibiotic era. Cold Spring Harb Perspect Med 2013 Apr 1;3(4):a010306

22. Bishop SM, Walker M, Rogers AA, Chen WY. Importance of moisture balance at the wound-dressing interface. J Wound Care 2003; 12(4):125-8

23. Martin M. Physiology of Wound Healing. In: Flanagan M, Martin M. Wound Healing and Skin Integrity: Principles and Practice. Wiley-Blackwell 2013

24. Schultz GS, Sibbald RG, Falanga V et al. Wound bed preparation: a systematic approach to wound management. Wound Repair Regen 2003;11(Suppl 1):S1-28

25. Panuncialman J, Falanga V. The science of wound bed preparation. Surg Clin North Am 2009 Jun;89(3):611-26. doi 10.1016/j.suc. 2009.03.009

26. European Wound Management Association (EWMA). Position Document. Wound bed preparation in practice. London, MEP Ltd 2004

27. D'Atanasio N, Capezzone de Joannon A, Mangano G et al 
A new acid-oxidizin solution: assessment of its role on MethicillinResistant Staphilococcus aureus (MRSA). Biofilm morphological changes. Wounds 2015;27:265-73

28. McDonnell G, Russell AD. Antiseptics and disinfectants: activity, action, and resistance. Clin Microbiol Rev 1999 Jan;12(1):147-79

29. D'Atanasio N, Capezzone de Joannon A, Mangano G et al. Ruolo di una nuova soluzione acido-ossidante nel controllare il microambiente delle ferite. Gazzetta Medica Italiana Archivio per le Scienze Mediche 2015 Ott;174(10):431-9

30. Sibbald RG, Williamson D, Orsted HL et al. Preparing the wound bed--debridement, bacterial balance and moisture balance. Ostomy Wound Management 2000;46:14-35

31. Kerstein MD. The scientific basis of healing. Adv Wound Care 1997;10(3):30-6

32. Lo SF, Hayter M, Hu WY et al. Symptom burden and quality of life in patients with malignant fungating wounds. J Adv Nurs 2012 Jun;68(6):1312-21

33. Hareendran A, Bradbury A, Budd J et al. Measuring the impact of venous leg ulcers on quality of life. J Wound Care 2005 Feb;14(2):53-7 